\title{
Large-Field Visual Motion Directly Induces an Involuntary Rapid Manual Following Response
}

\author{
Naoki Saijo, Ikuya Murakami, Shin'ya Nishida, and Hiroaki Gomi \\ NTT Communication Science Laboratories, Nippon Telegraph and Telephone Corporation, Kanagawa 243-0198, Japan
}

\begin{abstract}
Recent neuroscience studies have been concerned with how aimed movements are generated on the basis of target localization. However, visual information from the surroundings as well as from the target can influence arm motor control, in a manner similar to known effects in postural and ocular motor control. Here, we show an ultra-fast manual motor response directly induced by a large-field visual motion. This rapid response aided reaction when the subject moved his hand in the direction of visual motion, suggesting assistive visually evoked manual control during postural movement. The latency of muscle activity generating this response was as short as that of the ocular following responses to the visual motion. Abrupt visual motion entrained arm movement without affecting perceptual target localization, and the degrees of motion coherence and speed of the visual stimulus modulated this arm response. This visuomotor behavior was still observed when the visual motion was confined to the "follow-through" phase of a hitting movement, in which no target existed. An analysis of the arm movements suggests that the hitting follow through made by the subject is not a part of a reaching movement. Moreover, the arm response was systematically modulated by hand bias forces, suggesting that it results from a reflexive control mechanism. We therefore propose that its mechanism is radically distinct from motor control for aimed movements to a target. Rather, in an analogy with reflexive eye movement stabilizing a retinal image, we consider that this mechanism regulates arm movements in parallel with voluntary motor control.
\end{abstract}

Key words: involuntary visuomotor response; reflexive manual response; large-field visual motion; reaching arm movement; random-dot kinematogram; ocular following response

\section{Introduction}

Several motor control studies have discussed how the CNS generates motor commands for aimed movements to a target (Prablanc et al., 1979; Ghez and Krakauer, 1991; Georgopoulos, 1995; Kawato, 1999; Wolpert and Ghahramani, 2000). Computational models postulate distinct processes for trajectory planning, coordinate transformation (kinematics), and motor command generation (dynamics). In line with these computational requisites, many neurophysiological studies have examined whether these processes are sequentially solved in a series of hierarchically connected brain regions (Alexander and Crutcher, 1990; Caminiti et al., 1992; Kalaska et al., 1992; Kakei et al., 1999). Previous studies (Kawato, 1999; Wolpert and Ghahramani, 2000) emphasized that feedforward calculation by inverse models and prediction by forward models may both contribute to generating motor commands for aimed movement. The sequence of several distinct computational processes and considerable feedback delay in the nervous system imply that mechanisms of voluntary arm motor control operate quite slowly.

In contrast to such elaborate but slow voluntary control, fast

Received 0ct. 6, 2004; revised March 26, 2005; accepted April 7, 2005

We thank P. Haggard for improving this manuscript and T. Hirahara and K. Moriya for advice and support.

Correspondence should be addressed to Hiroaki Gomi, NTT Communication Science Laboratories, Nippon Telegraph and Telephone Corporation, Wakamiya 3-1, Morinosato, Atsugi, Kanagawa 243-0198, Japan. E-mail: gomi@idea.brl.ntt.co.jp.

D01:10.1523/JNEUROSCI.4143-04.2005

Copyright $\odot 2005$ Society for Neuroscience $\quad$ 0270-6474/05/254941-11\$15.00/0 visuomotor control exists in several motor systems. For example, large-field visual motion induces leg muscle activation with very short latency for stabilizing posture (Nashner and Berthoz, 1978) and induces smooth eye movements [ocular following response (OFR)] for stabilizing retinal images (Kawano and Miles, 1986; Miles et al., 1986; Gellman et al., 1990). Studies of the neural mechanism of the OFR (Kawano et al., 1992, 1994; Shidara et al., 1993; Gomi et al., 1998; Takemura et al., 2001) indicate that it is reflexively driven by large-field visual motion and is mediated by a short pathway distinct from those for other, longer-latency types of eye movements. Similar to the oculomotor task, humans often make accurate arm reaching movements during dramatic body movements accompanied by visual motion, for example when a rugby player catches a ball while being pushed by another player. It is known that the vestibular system is insufficient in compensating body motion (Blouin et al., 1995, 1998). Do we have a fast motor regulation mechanism for arm movement when the visual world moves? And if so, what mechanism is used to generate fast responses?

Here, we report an ultra-fast manual response to a large-field visual motion, which is analogous to the OFR. A series of results indicate that this response is directly driven by large-field visual motion signals through a reflexive pathway rather than by a shift in the position of target representation (Goodale et al., 1986; Prablanc and Martin, 1992; Brenner and Smeets, 1997; Pisella et al., 1998, 2000; Day and Lyon, 2000; Desmurget and Grafton, 2000; Brouwer et al., 2002; Whitney et al., 2003) or by the effect of 
ocular movement (Henriques et al., 1998, 2003; Soechting et al., 2001). Our findings suggest a new computational framework for understanding the control mechanism of arm movement.

\section{Materials and Methods}

\section{Experimental setup and data analysis}

Each subject sat in front of the manipulandum while strapped securely to the chair back, and his head was placed on a chin support as shown in Figure 1 . The right forearm was tightly coupled with the handle by a molded plastic cuff and supported in the vertical direction by a beam. The hand was able to move freely in any direction within the horizontal plane at shoulder level. The hand position was obtained from the manipulandum system (Gomi and Kawato, 1996) at $500 \mathrm{~Hz}$.

Visual stimuli were generated by a computer and presented via a data projector (refresh rate, $60 \mathrm{~Hz}$; PLUS U2-X2000; PLUS Vision Corp., Tokyo, Japan $)$ on a horizontal screen $(1.2 \times 1.0 \mathrm{~m})$ placed just above the subject's forearm (see Fig. 1). The screen concealed the arm from the subject's view. As a result of the frame asynchrony of the projector, the actual visual stimulus (motion) could start 24.7-41.7 ms after the stimulus trigger signal (see below, Experimental protocol). To counteract these fluctuating delays, the actual time of stimulus onset in each trial was optically monitored (at $6 \mathrm{kHz}$ ) with a photodiode (S1223-1; Hamamatsu Photonics, Hamamatsu, Japan). All of the data in the offline analysis were temporally aligned at the actual visual motion onset.

In experiment 1 , surface electromyograms (EMGs) of shoulder and elbow single-joint muscles (pectoralis major, deltoid posterior, brachioradialis, and triceps lateral) and cross-joint muscles (biceps and triceps long-head) were obtained with differential Ag-AgCl electrodes, then amplified, filtered (bandpass, $30-1.5 \mathrm{kHz}$ ), and digitally recorded at $3 \mathrm{kHz}$. The EMGs were then rectified, smoothed (20 ms moving average, causal filtering), and normalized by the maximum EMG of the corresponding muscle during static force regulation tasks ( $10 \mathrm{~N} ; 16$ radial directions) [called rectified, smoothed, and normalized EMG (rEMG)]. For two subjects in experiment 1, we also recorded the EMGs of the cervical and back muscles (sternocleidomastoid and erector spinas) demonstrated to be involved in trunk and neck postural adjustment (Keshner et al., 1988; Henry et al., 1998) and then rectified and temporally averaged EMGs $(100-150 \mathrm{~ms})$ to check the condition-dependent EMG changes. Note that we confirmed in advance that these muscles were activated during postural adjustments of the trunk and neck, respectively. Latencies of hand acceleration and rEMG were detected by pooled successive sample $t$ tests (5\%) between trials involving rightward and leftward visual motion. In supplemental experiments of experiment 1, we also recorded right eye movements $(500 \mathrm{~Hz}$; no-filter mode; EyeLink II; SR Research, Osgoode, Ontario, Canada) to compare the latencies of manual and ocular responses to stimuli. The latency of ocular velocity response was also detected by successive $t$ tests (5\%) after excluding the trials with saccadic intrusion.

Eight subjects (20-27 years of age) participated in experiments 1 and 2 , three subjects (24-30 years of age) in experiment 3 , four subjects (22-27 years of age) in experiment 4 , five subjects (20-30 years of age) in experiment 5 , four subjects (20-27 years of age) in experiment 6 , and seven subjects (20-30 years of age) in experiment 7 . In the two supplemental experiments of experiment 1 , three subjects (21-30 years of age) for the first experiment and three subjects (24-31 years of age) for the second experiment participated. All were male. No subjects had ever experienced any visual or motor deficits, and all gave informed consent to participate in the study, which was approved by the NTT Communication Science Laboratories Research Ethics Committee.

\section{Experimental protocol}

Experiment 1. The visual stimulus was a dense random-dot pattern occupying the full screen. Each pixel $(1.7 \times 1.7 \mathrm{~cm})$ was either black or white with $50 \%$ probability. Before each trial, the pattern remained stationary, accompanied by a start marker (a blue circle of $3 \mathrm{~cm}$ diameter), a target marker (a red disk of $3 \mathrm{~cm}$ diameter), and a fixation spot $(1 \times 1$ $\mathrm{cm})$ at $(0,0.35),(0,0.55)$, and $(-0.17,0.45)$, respectively [where the $(x, y)$ indicates the $x$ (in meters) rightward and $y$ (in meters) frontal direction relative to the shoulder position on the screen plane]. The subject initially placed his hand at the start position using a marker indicating current hand position. This marker was extinguished before starting each trial. The subject then moved his hand from the start to the target with the instructed duration $(1.0 \mathrm{~s})$, which was given by a series of beeping sounds. When the hand had traveled $3 \mathrm{~cm}$ from the start, a trigger signal was sent to the visual stimulus generator. The trigger signal turned off the start and target markers and the fixation spot and moved the pattern at 0.7 $\mathrm{m} / \mathrm{s}$. The subject was also asked not to move and not to close his eyes after eliminating the fixation spot. Of 100 repeated trials, the pattern moved rightward (positive along the $x$-axis) in 30 trials, moved leftward (negative along the $x$-axis) in 30 trials, or remained stationary, in random order. For all of the conditions, the pattern disappeared $0.5 \mathrm{~s}$ after the trigger.

To check whether the occurrence of OFR is a prerequisite for the occurrence of manual following response (MFR), we did two supplemental experiments. In the first one, the movement and EMG of the right arm and the movement of the right eye were recorded simultaneously while the subjects $(n=3)$ performed the same task as in experiment 1 . In the second supplemental experiment, we compared the magnitude changes in the OFR and the MFR under three stimulus conditions for new subjects $(n=3)$. The first condition was equivalent to the original condition of the first experiment, namely, a full-field visual motion stimulus, with the fixation spot eliminated during the visual motion (FN condition). In the second condition, the fixation spot was not eliminated but remained at the same position $[(-0.17,0.45) \mathrm{m}]$ during the full-field visual motion (FF condition). In the third condition, the stimulus size was halved: the moving random-dot pattern was shown only in the righthalf field ( $x$ greater than $-0.08 \mathrm{~m}$ ) on the screen (HF condition). The remaining left-half screen was uniformly black except for the fixation spot shown at the same position. The same arm movement was requested as in the first experiment. As in the original experiment, the checker pattern suddenly moved either to the left or to the right during arm movement in 30 of 100 trials (random order) for each direction. Again, eye movements and hand movements of the subject were monitored concurrently.

Experiment 2. Before each trial, three possible targets, namely the original target and two additional markers presented $5 \mathrm{~cm}$ to the left and right of the original one, were shown on the screen together with the random dot pattern. At the beginning of each session, the subject was instructed to make either a "proreaction" or "antireaction." In the former case, he had to reach for the target on the same side as the direction of visual motion; in the latter case, he had to reach for the target on the opposite side. If the visual stimulus did not move, the subject had to reach for the original target. The subject was instructed to perform these voluntary reactions as soon as possible after motion direction was detected. The left, right, and original targets disappeared at the same time before starting the visual motion as in experiment 1 .

Experiment 3. We used a sparse random-dot pattern having shortlifetime coherent motion (Newsome and Pare, 1988) as the visual stimulus. In this stimulus, a given proportion of dots moved in the same direction, while the other dots moved in random directions, with each dot having a lifetime of only two frames. Although the dot size and the motion speed were the same as the original random-dot motion, the dot density was much lower $\left(1723 \mathrm{dots} / \mathrm{m}^{2}\right)$. The motion coherence (the proportion of coherently moving dots) was set at 50, 30, and $10 \%$ in separate sessions (see Fig. 7A). The experimental protocol was the same as in experiment 1 , except that $0.5 \mathrm{~s}$ before the "go" beeping sound, the position markers (start, target, and fixation) were turned off to exclude the possibility of any positional cues interacting with the moving stimulus.

Experiment 4 . The subject viewed a visual stimulus without moving his hand. The stimulus sequence was the same as that in experiment 1 , except that the target marker was randomly placed at one of five locations $(x=$ $-0.02,-0.01,0,0.01$, or $0.02 \mathrm{~m} ; y=0.55 \mathrm{~m})$. After the stimulus presentation, the subject was asked to report the remembered target position (along the $x$-axis) by using the mouse cursor, which could be moved only along a visible transversal line on the screen.

Experiment 5. We investigated whether the quick response occurs during targetless arm movement as well. The subject was asked to perform 
three tasks in separate sessions. In the first task [hitting $(\mathrm{H})$ task] (see Fig. $8 \mathrm{~B}$ ), the subject was asked to start moving his hand at the go beeping sound, hit a rectangular target marker, and then make a follow-through movement beyond the target location, in the most natural direction. The stop position of the movement was not specified. The subject was also asked to keep watching the location of the hitting target during the movement (even after it disappeared). The hand start position was $(-0.13$, $0.30) \mathrm{m}$, and the target was displayed at one of the three positions at random $1.0 \mathrm{~s}$ before the go beeping sound: center $[(-0.13,0.38) \mathrm{m}]$ in 60 trials, left $[(-0.16,0.38) \mathrm{m}]$ in 20 trials, and right $[(-0.10,0.38) \mathrm{m}]$ in 20 trials. The selected target was extinguished when the hand had traveled 8 $\mathrm{cm}$ in the $y$ direction from the start position. Success was immediately given by a beeping sound if the hand had correctly passed the hitting target position $(1 \times 1 \mathrm{~cm}$ square $)$ within $0.3-0.7 \mathrm{~s}$ after the go signal. In other words, the subject knew he failed in hitting the target by the absence of this sound. The same random-dot pattern was shown on the screen. It was moved rightward or leftward $47 \pm 17 \mathrm{~ms}$ after the hand had gone beyond the target location in 40 of 60 center-target trials and remained stationary in the other trials. In either case, the visual stimulus was turned off $0.5 \mathrm{~s}$ after passing target location.

In the second and third tasks, to compare the pattern of armmovement variability between the hitting and reaching tasks, the subject was asked to reach his hand from the same start position for a target (instructed movement duration, $1 \mathrm{~s}$ ). In the second task, the target was placed at $(-0.13,0.55) \mathrm{m}$ [fixed-target reaching $(\mathrm{R})$ task] (see Fig. $8 B$ ), and in the third task [variable-target reaching (R2) task] (see Fig. $8 \mathrm{~B}$ ), the target was varied randomly from trial to trial, and its position was sampled from the Gaussian distribution characterized by the mean and variance of the ends of movement in the hitting task of the corresponding subject. The movement end was identified as a tangential velocity threshold of $<0.01 \mathrm{~m} / \mathrm{s}$. In the last two tasks, the target was extinguished at the go beeping sound. The background visual stimulus was a stationary random-dot pattern and was turned off $0.5 \mathrm{~s}$ after the hand passed a particular $y$-directional position $(y=0.38 \mathrm{~m})$ as in the first task. Twenty trials were run for each of the two tasks.

Experiment 6. A constant bias force was continuously applied to the handle by the manipulandum. There were seven force conditions: $0,-6$, and $-9 \mathrm{~N}$ in the $y$ direction; $-9,-6,6$, and $9 \mathrm{~N}$ in the $x$ direction. In addition to the standard task instruction, the subject was requested to make the trajectory as straight as possible. The protocol and visual stimuli were exactly the same as in experiment 1.

Experiment 7. We tested two stimulus speeds, 0.7 and $0.07 \mathrm{~m} / \mathrm{s}$, in separate experimental sets. The experimental protocol was exactly the same as in experiment 1 except for the number of trials ( 18 for rightward, 18 for leftward, and 24 for control).

\section{Results}

Involuntary and rapid manual responses elicited by large-

field visual motion

In the first experiment, we examined whether large-field visual motion has any effect on arm movement. Subjects made reaching movements with their right hand from proximal to distal positions in the horizontal plane at shoulder level, using a manipulandum (Gomi and Kawato, 1996). The arm was occluded from view by a screen on which visual stimuli were projected. At the beginning of each trial, the screen was filled with a stationary random-dot pattern (Fig. 1). When the hand had moved $5 \mathrm{~cm}$ from the start position (Fig. $2 \mathrm{~A}$, marked on the trajectories), target and fixation points were eliminated, and the dot pattern began to move rightward or leftward at random with a constant speed.

The hand acceleration along the $x$-axis changed shortly after the visual-motion onset (Fig. $2 B$ ), significantly deviating from the control condition in which no visual motion was presented. The latency of these responses to visual motion was $126 \pm 20 \mathrm{~ms}$. (This notation, meaning "intersubject mean $\pm 1 \mathrm{SD}$," will be consistently used hereafter to express the latency; for the detec-
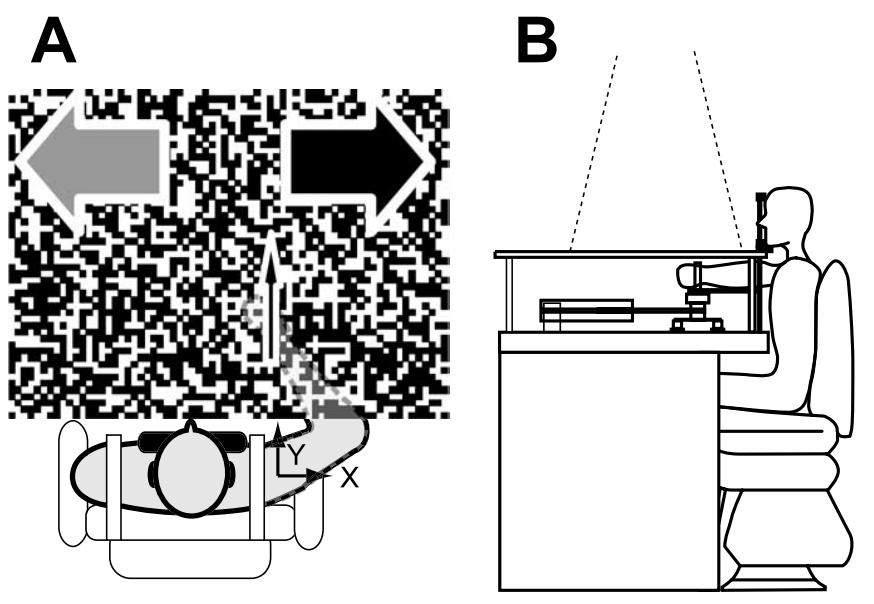

Figure 1. Experimental setup. A, Top view. The subject's forearm was occluded by the screen, on which a random dot pattern was projected. That pattern moved rightward (black arrow) or leftward (gray arrow) during the arm extension. The center thin arrow denotes the hand movement direction. The origin of the special axes is located at the shoulder joint. $\boldsymbol{B}$, Side view. The subject's forearm was fixed by a molded plastic cuff to the supporting beam. The subject's chin was placed on the supporting device. The visual stimulus was projected from the ceiling onto the horizontal screen.

tion method, see Materials and Methods.) This additional component of the hand movement was in the same direction as the visual motion. By analogy with the short-latency eye movement induced by a sudden large-field visual motion, called the OFR (Miles et al., 1986; Gellman et al., 1990), we use the term MFR to describe this behavior.

rEMGs revealed the specificity of the MFR. The rEMG increased in the shoulder flexor and decreased in the elbow extensor following the visual motion to the left (Fig. 2C, gray lines), whereas it increased in the elbow extensor following the visual motion to the right (Fig. 2C, black lines). The EMG responses occurred with short latencies (shoulder flexor, $98 \pm 13$ ms; elbow extensor, $104 \pm 14 \mathrm{~ms}$ ) after the visual-motion onset. Importantly, EMGs of the cervical and back muscles did not differ after leftward and rightward visual motions ( $t$ test; $p>0.05$ ), whereas the EMGs of arm muscles simultaneously recorded significantly depended on the visual motion direction (Table 1). Therefore, the MFR resulted from specific arm muscle activation driven by visual motion, not from visually induced generalized postural stabilization of the neck and trunk (Nashner and Berthoz, 1978).

Because interaction between the eye and arm movements has been suggested previously (Henriques et al., 1998, 2003; Soechting et al., 2001; Ariff et al., 2002), we also investigated the temporal relationship between the OFR and MFR. Note that, because of the greater inertia of the arm and different force production dynamics of the eye and arm muscles (Ito et al., 2004), eye and arm kinematic data cannot be compared directly. Instead, to examine a possible signal flow from the eye somatosensory signal to the arm motor command, we compared the latencies of the arm EMG response driving the MFR with the latency of the OFR. They were simultaneously recorded in the arm movement task. Figure 3 shows an example of temporal responses of the shoulder flexor muscle rEMG (Fig. 3A) and the right-eye horizontal position (Fig. 3B, top) and velocity (Fig. 3B, bottom) for a typical subject. The eye smoothly moved in the direction of the visual stimulus, as observed in studies of the OFR in a comparable paradigm (Miles and Kawano, 1986; Masson et al., 2000). For this subject, the latency of the shoulder flexor muscle EMG response ( $85 \mathrm{~ms}$ ) was comparable with that of the OFR (86 ms). 
A

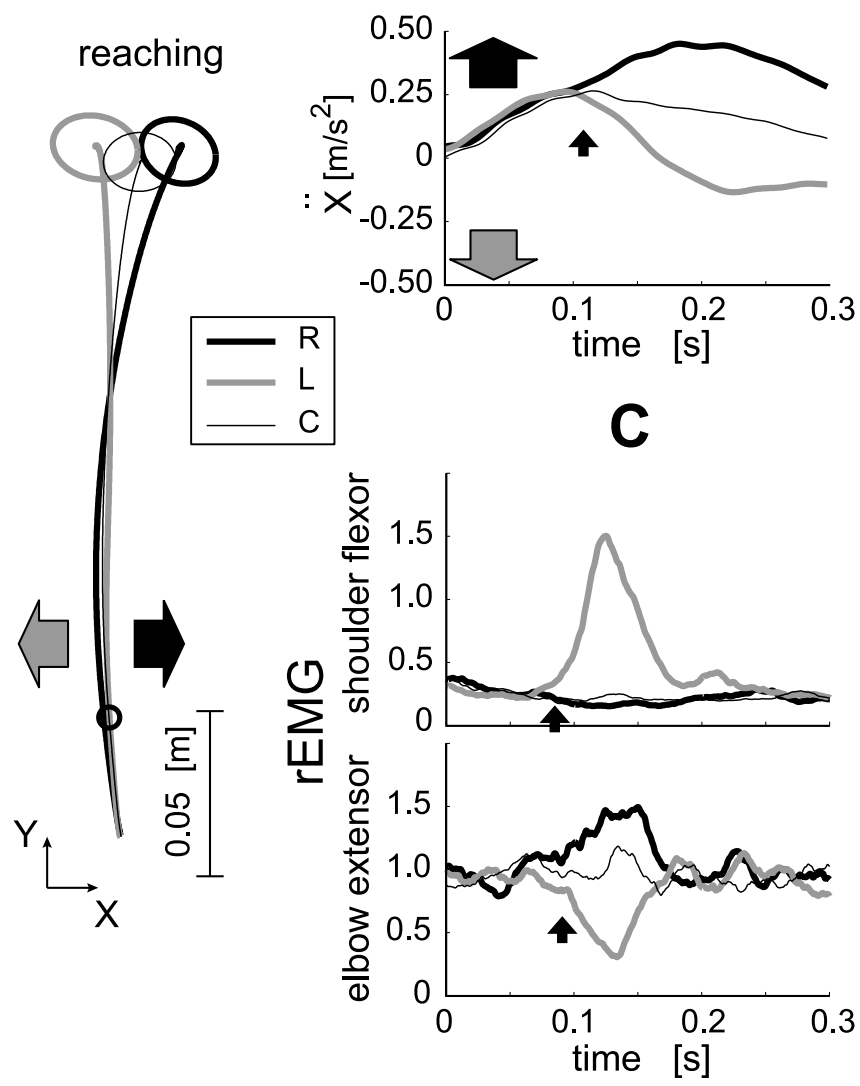

Figure 2. Short-latency manual response caused by visual motion. $A$, Averaged hand movement trajectories of a typical subject in the three visual stimulus conditions ( 30 trials each for the left and right, 40 trials for the control). Thin black, thick black, and thick gray lines indicate the control (C; no visual motion), rightward $(\mathrm{R})$, and leftward $(\mathrm{L})$ visual-motion conditions, respectively. Arrows denote the visual-motion directions (black, rightward; gray, leftward). Ellipses at the movement ends indicate the SD of the arrival positions in each condition, and the small circle on the trajectory indicates hand position at the time the stimulus started to move. $\boldsymbol{B}$, Temporal patterns of averaged $x$ acceleration (the direction orthogonal to the movement) in the three conditions. Time 0 corresponds to visual motion onset. The response latency (filled small arrow) in this example was $110 \mathrm{~ms}$. C, Temporal patterns of the rEMG of the shoulder flexor and elbow extensor single-joint muscles. The latencies of the EMG responses (arrows) were $85 \mathrm{~ms}$ (shoulder flexor) and $91 \mathrm{~ms}$ (elbow extensor).

Table 1. Muscle activity changes after visual motion stimuli (temporal mean of rEMG for the interval of $100-150$ ms after the stimulus onset)

\begin{tabular}{lllllll}
\hline & \multicolumn{7}{l}{ Muscle EMG $(\mu \mathrm{V})$} & & & \\
\cline { 2 - 7 } Subject (stimulus direction) & Shoulder flexor & Elbow extensor & Neck left & Neck right & Back left & Back right \\
\hline A & & & & & & \\
(R) & $3.1(1.7)^{*}$ & $31.0(10.2)^{*}$ & $1.7(0.6)$ & $1.3(0.5)$ & $1.2(0.6)$ & $0.9(0.8)$ \\
(L) & $14.3(8.8)$ & $20.4(7.2)$ & $1.7(0.4)$ & $1.4(0.8)$ & $1.2(0.6)$ & $0.7(0.5)$ \\
B & & & & & & \\
(R) & $5.3(2.6)^{*}$ & $17.8(4.0)^{*}$ & $1.3(0.6)$ & $2.7(1.3)$ & $0.8(0.3)$ & $1.1(0.4)$ \\
(L) & $11.8(3.0)$ & $12.1(4.4)$ & $1.2(0.6)$ & $2.4(1.2)$ & $1.1(0.9)$ & $1.3(0.8)$ \\
\hline
\end{tabular}

The values in parentheses denote SDs of trial variance $(n=30)$. Asterisks denote statistical significance $(t$ test; $p<0.05)$ of the difference between the responses in the leftward $(\mathrm{L})$ and rightward $(\mathrm{R})$ stimulus conditions.

For the other two subjects, these latencies were 88 and $93 \mathrm{~ms}$ for the shoulder muscle rEMG and 87 and $89 \mathrm{~ms}$ for the OFR, respectively. Considering the transmission time from the motor cortex to the arm muscle and the OFR command generation process (see Discussion), this result suggests that the MFR cannot simply be attributed to OFR by some form of motor entrainment.

In addition, the dissociation in the magnitude changes in the
OFR and MFR by continuous presentation of the fixation spot and stimulus-size reduction further indicates that the MFR is not always linked to the OFR. Figure 4 shows the amplitudes of the MFR and OFR under three stimulus conditions: FN condition, FF condition, and HF condition. Without the fixation spot, the effect of visual motion was statistically significant for all subjects both in the OFR and MFR. By introducing the fixation spot, the OFR amplitude was reduced but not completely suppressed (significant for two of the three subjects). This remaining of the OFR may be a result of the presence of the strong motion stimulus. When we further reduced the visual stimulus size to the right half of the screen, the OFR completely disappeared (insignificant for all subjects). In contrast, the MFR amplitudes for the rightward and leftward motion stimuli were significantly different in all conditions for all three subjects. Namely, MFR was robustly observed in each condition, regardless of the occurrence of OFR. Therefore, we conclude that the occurrence of OFR is not a prerequisite for the occurrence of MFR.

In the next experiment, we examined whether these responses were voluntary or reflexive (automatic) reactions. We asked the subjects to react in the same (proreaction) or opposite (antireaction) direction to that of the visual motion as soon as they identified its direction. As shown in Figure $5 A$, the hand quickly curved to the left or right in the proreaction task, whereas the opposite reaction was greatly delayed in the antireaction task. From the initial phases of the hand $x$ acceleration in the antireaction task shown in Figure 5B, it is clear that the hand was initially accelerated in the same direction as the visual motion, followed by the opposite (instructed) directional acceleration. The same characteristics are also evident in the rEMG data. In the proreaction (solid lines), the initial activations in rEMGs shown in Figure $5 C$ were subsequently maintained. In the antireaction (dash-dot lines), virtually the same muscle activations as in the proreaction occurred first, followed by different muscle activation patterns. Acceleration latencies ( $122 \pm 20 \mathrm{~ms}$ for the proreaction; $127 \pm 16$ $\mathrm{ms}$ for the antireaction) and EMG latencies (shoulder, $92 \pm 7 \mathrm{~ms}$ for the proreaction, $94 \pm 8 \mathrm{~ms}$ for the antireaction; elbow, $102 \pm$ $14 \mathrm{~ms}$ for the proreaction, $97 \pm 14 \mathrm{~ms}$ for the antireaction) suggested that the initial phases of the responses both for the proreaction and antireaction were similar to those in the simple reaching movement (Fig. 2C). The assistive quick response in the proreaction suggests a functional interaction between the MFR and voluntary movement. The quick response in the direction of visual motion despite the antireaction instruction indicates that the MFR cannot be voluntarily suppressed, suggesting a reflex mechanism for MFR (Marsden et al., 1972).

Furthermore, the MFR was modulated not only by the stimulus direction but also by the speed of visual motion. Figure $6 \mathrm{~A}$ shows typical temporal patterns of the MFR induced by two speeds of visual motion in rightward and leftward directions ( \pm 0.7 and $\pm 0.07 \mathrm{~m} / \mathrm{s}$ ) in experiment 7 . As shown in this figure, the response amplitude for the faster stimulus $(0.7 \mathrm{~m} / \mathrm{s})$ was greater than that for the slower one $(0.07 \mathrm{~m} / \mathrm{s})$. To quantify the response amplitudes at different stimulus speeds, we introduce an index, the "acceleration modulation depth," defined as the temporal average of the difference in $x$ acceleration between the rightward and leftward motion conditions for a period of 150-200 ms (Fig. 6A, shaded time range). As observed in 

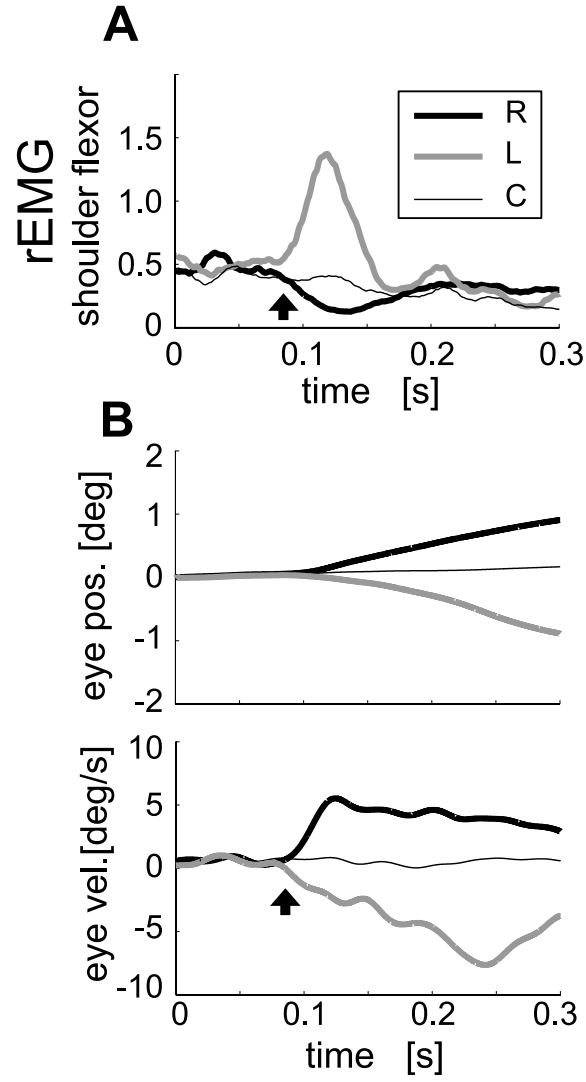

Figure 3. Arm muscle responses and ocular responses induced by large-field visual motions (simultaneously recorded). $\boldsymbol{A}$, Mean rEMG responses of shoulder flexor muscle induced by the rightward ( $R$; thick black), leftward (L; thick gray), and control (C; thin black) visual stimuli. Detected latency (arrow) by successive $t$ tests was $85 \mathrm{~ms}$. B, Mean ocular responses [top graph, position (pos.); bottom graph, velocity (vel.)] of the right eye during this task. Detected latency (arrow) by the successive $t$ tests for the velocity was $86 \mathrm{~ms}$. (Because of saccadic intrusion, 4 trials of 100 were excluded for averaging and detecting latency.) deg, Degrees.

the temporal patterns, the difference in the acceleration modulation depths was observed in all subjects as shown in Figure $6 B$ and was statistically significant (paired $t$ test; $p<0.01$ ).

\section{The MFR occurs without positional information in the visual stimulus}

What information in the stimulus induces the MFR? Considering that random-dot motion has been successfully used in vision studies to tap the brain mechanisms specific to visual motion analysis (Braddick, 1980; Nakayama and Tyler, 1981; van de Grind et al., 1986), it is argued that positional cues in a randomdot pattern would be scarcely accessible by the visual system. Thus, it is likely that large-field motion information also induces the MFR. To obtain specific evidence on this point, we reduced the positional information in the stimulus by using short-lifetime coherent motions (Newsome and Pare, 1988). The motion coherence (the proportion of dots moving in the same direction) was set at 50,30, and $10 \%$ in separate sessions (Fig. 7A) (for details, see Materials and Methods, Experimental protocol, Experiment 3). The top panel of Figure $7 B$ shows the hand acceleration responses (plotted as the deviation from the control condition) to the rightward and leftward visual motions of $50 \%$ coherence patterns. Similar to the original experiments, shortlatency acceleration responses were observed (acceleration latency, $153 \pm 20 \mathrm{~ms}$ ). When visual motion coherence was reduced to 30 and $10 \%$, the amplitude of the hand acceleration responses full field

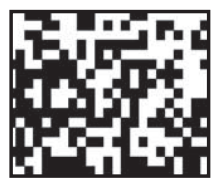

\section{half field}

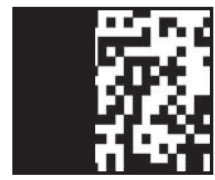

Subj. A

FN FF HF
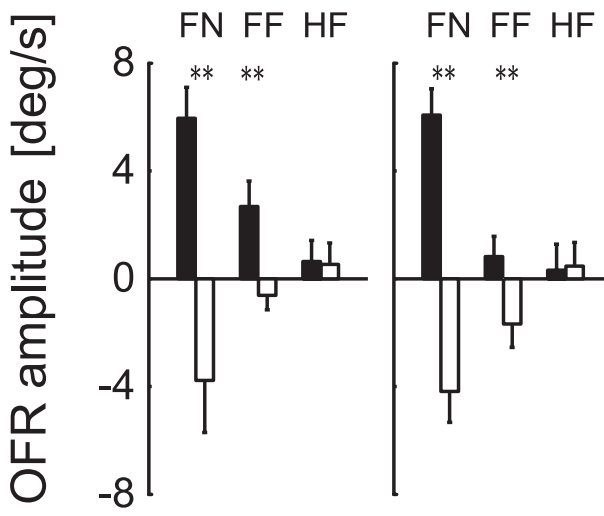

Subj. B Subj. C

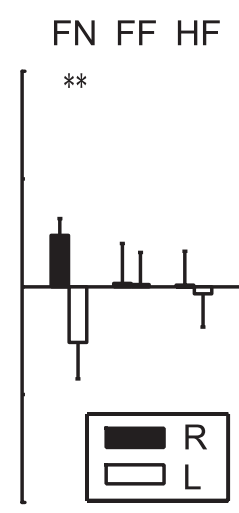

0.2
0.1
0
0.1
-0.2

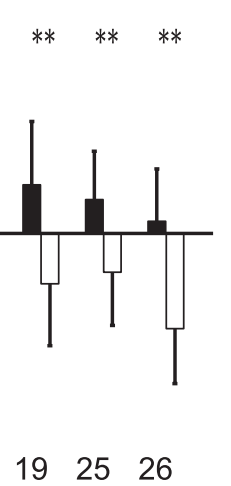

$\begin{array}{lll}19 & 26 & 22\end{array}$

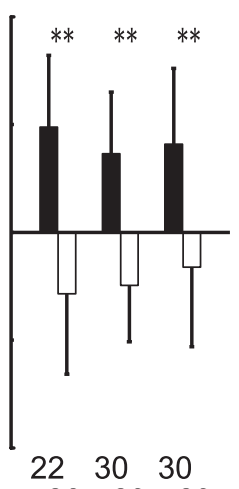

$\begin{array}{lll}26 & 29 & 29\end{array}$

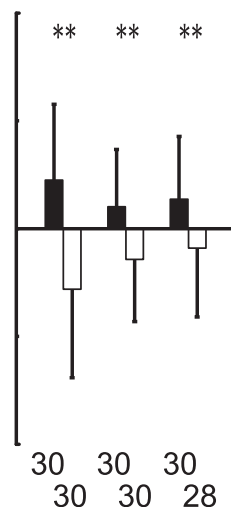

$\begin{array}{lll}30 & 30 \quad 28\end{array}$
Figure 4. Amplitude changes in the OFR and MFR by fixation spot and stimulus reduction. The top diagram represents the full- and half-field visual stimuli displayed on the screen (for details, see Materials and Methods). The top three panels show the OFR amplitudes (temporal mean ocular velocity between 150 and $200 \mathrm{~ms}$ after the stimulus onset) for three subjects (Subj.) for the rightward ( $R$; filled bar) and leftward ( $L$; open bar) visual motions in three conditions: FN, FF, and HF. The bottom three panels show the MFR amplitudes (mean hand acceleration change from the control response in the $x$ direction between 150 and $200 \mathrm{~ms}$ after the stimulus onset) for the same three subjects recorded simultaneously with the ocular movement. Each error bar denotes intertrial variation (SD) in each condition. The trials with saccadic intrusion were excluded from both ocular and arm movement data before averaging. Each number placed at the bottom of the graph denotes the number of trials for averaging and statistical tests. The double asterisks above the bar-pair denote the statistical significance ( $p<$ 0.01) of the difference between these mean amplitudes. deg, Degrees.

decreased. To quantify the response amplitudes in different motion coherences, we again use the acceleration modulation depth. All subjects in this experiment exhibited a positive relationship between the visual motion coherence percentage and the acceleration modulation depth (Fig. 7C). These results clearly show that the degree of visual motion signals in a large field affected the MFR, even when positional cues were effectively absent.

\section{Effects of visual motion on target representation}

Recently, in parallel with our study, a few studies (Brenner and Smeets, 1997; Whitney et al., 2003) have reported rapid arm re- 
sponses driven by fields of visual motion as in the case of the MFR. These studies offered an interpretation that the visual motion drives the arm movements indirectly by shifting the position of the reaching target represented in the brain. Because visual motion can sometimes affect the perceived position of a visual object nearby (Ramachandran, 1987; Murakami and Shimojo, 1993; Nishida and Johnston, 1999; Whitney and Cavanagh, 2000), such a target shift might trigger the quick arm adjustment. The previous experiments (Brenner and Smeets, 1997; Whitney et al., 2003), however, did not distinguish between an effect of large-field visual motion on target localization and a "direct" effect of visual field motion on arm movement. Given that there are a number of differences in the experimental condition, it is debatable to what extent the rapid manual responses reported in these studies shared the underlying mechanism with the MFR.

In the next experiment (experiment 4), we therefore examined whether the MFR is accompanied by any shift in the internally represented target position. Subjects were asked to locate the mouse cursor at the remembered target position after the rightward or leftward moving pattern was presented (15 trials for each direction). Note that, unlike the previous studies (Brenner and Smeets, 1997; Whitney et al., 2003), the target was eliminated before starting visual motion. As shown in Table 2, the perceptual match to the target position was not significantly different in the two opposite directional visual motion conditions for any subject. In contrast, the mean movement end was significantly shifted ( $t$ test; $p<0.05$ ) by visual motion for six of eight subjects in experiment 1 . Note that the variances of perceived target positions in each visual condition (Table 2) were comparable with the variance of the movement-end positions (range of SD, 0.50$1.69 \mathrm{~cm}$ for the eight subjects in experiment 1).

\section{Target representation cannot explain an MFR during nonreaching movement}

Nevertheless, it could be argued that a separate representation of target location within the sensorimotor system (Goodale et al., 1986; Prablanc and Martin, 1992; Pisella et al., 1998, 2000; Day and Lyon, 2000; Desmurget and Grafton, 2000; Brouwer et al., 2002), as distinct from the representation of the perceived location, may be affected by large-field visual motion and used to drive the MFR. From that viewpoint, visual motion should only influence arm movements aimed at a (implicitly mislocalized) target. Thus, arm movements that are not aimed at any target location should not be influenced by visual motion. We therefore tested whether the MFR occurs during the follow-through phase of the movement after the target is hit (experiment 5), which is just like the follow-through of a tennis racket swing after the ball is hit. The subject in this experiment was instructed to move his hand toward the target (we will call this the hitting action) and let his hand continue to move in the most natural direction (we will call this the follow-through action). Note that the final hand position was irrelevant in this task, and visual motion began only
A

B anti-reaction
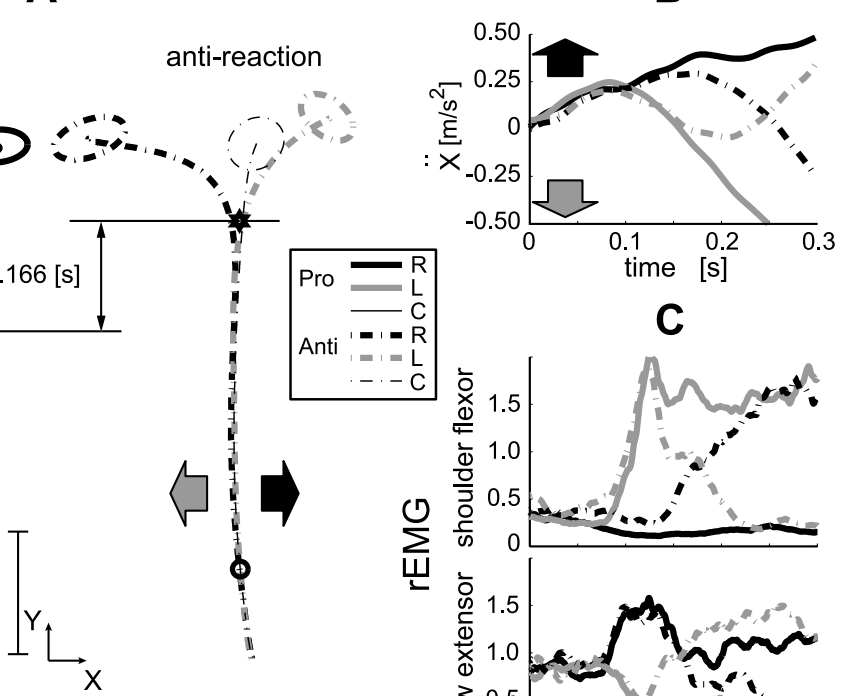

C

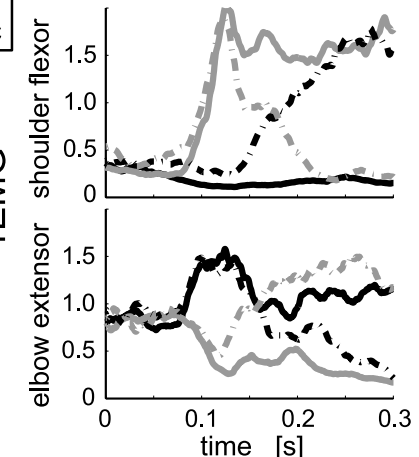

Figure 5. Manual responses in voluntary proreaction (Pro) and antireaction (Ant) tasks. $\boldsymbol{A}$, Averaged trajectories (30 trials each 作 curve represents the antireaction task. Black and gray arrows denote the stimulus directions (rightward and leftward,

after the hand passed the target location, at which time positional accuracy was no longer important for the subject. The target was randomly presented at one of the three positions (see Materials and Methods) (Fig. 8 B, $\mathrm{H}$ task) to encourage the subject to aim at the designated target and not to preplan the final position of the follow-through phase. Even in this condition, visual motion during the follow-through phase of arm movement elicited a clear MFR, as shown in Figure $8 \mathrm{~A}$. The mean latency and mean amplitude for the five subjects were $139 \pm 16 \mathrm{~ms}$ and $0.153 \pm 0.079$ $\mathrm{m} / \mathrm{s}^{2}$. These values were not significantly different ( $t$ test; latency, $p>0.2$; amplitude, $p>0.15$ ) from those in the simple reaching condition $\left(126 \pm 20 \mathrm{~ms}, 0.236 \pm 0.118 \mathrm{~m} / \mathrm{s}^{2}\right)$ in experiment 1 .

In addition, the follow-through trajectories showed a progressive increase in positional variability after the hand passed over the target location. This confirms that positional accuracy was not in fact important in the follow-through phase and abrogates the concern that some implicit target representation might exist at the end of the follow-through trajectory. Figure $8 B$ depicts mean trajectories (solid curve) and position variances (gray ellipses) during the following through after hitting the center target, the reach for a single distal target, and the reach for targets whose location was varied randomly from trial to trial with the positional variance of the movement end in the hitting task of the corresponding subject. The trajectory variance in the $x$ direction at the hitting distance (indicated by a dash-dot line) was not different between the $\mathrm{R}$ and the $\mathrm{H}$ tasks. Thereafter, trajectory variance increased progressively throughout the follow-through phase of the hitting task, suggesting a lack of precise positional control. In contrast, the increase of trajectory variability was smaller in the fixed-target reaching task. Accordingly, as shown in Figure $8 C$, positional variance in the $x$ direction at the end of the 

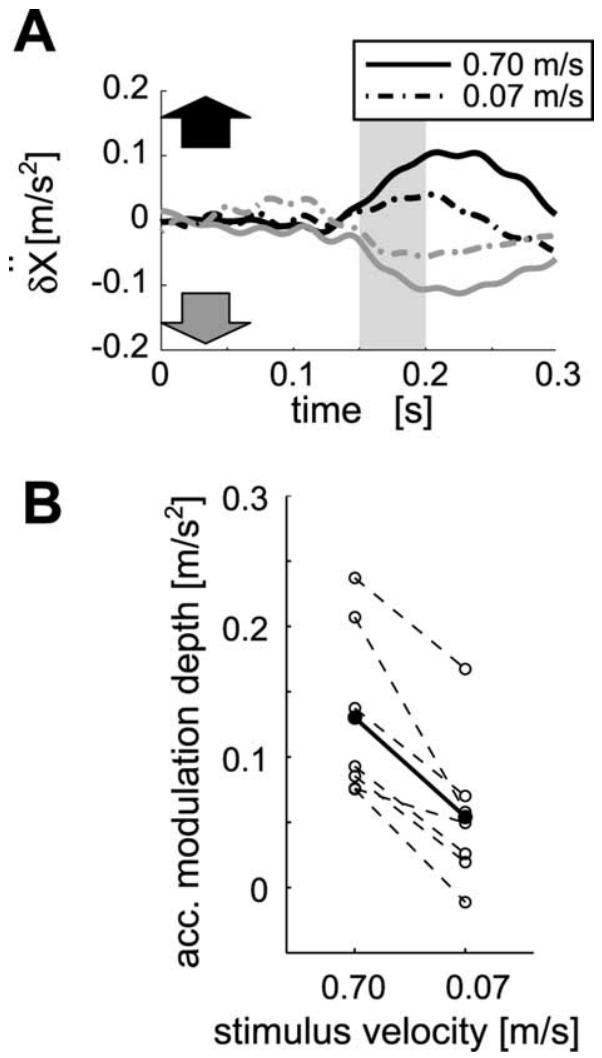

Figure 6. MFR modulation by visual stimulus speed. $\boldsymbol{A}$, Temporal patterns of $x$ acceleration induced by rightward (black) and leftward (gray) stimuli moving at $0.7 \mathrm{~m} / \mathrm{s}$ (solid line) and at $0.07 \mathrm{~m} / \mathrm{s}$ (dash-dot line) in a typical subject. The shaded area indicates the averaging interval for acceleration modulation depth (see Results). $\boldsymbol{B}$, Acceleration (acc.) modulation depth plotted against stimulus velocity. Each dashed line connects the data for each subject between the two velocity conditions, and the black line connects the data averaged over subjects. The mean response for the $0.7 \mathrm{~m} / \mathrm{s}$ stimulus was significantly grater ( $p<0.01$ by paired $t$ test) than that for the $0.07 \mathrm{~m} / \mathrm{s}$.

follow-through phase in the hitting task was significantly larger (intersubject paired $t$ test; $p<0.01$ ) than when reaching a remembered target at a comparable workspace location in the fixed-target reaching task, whereas positional variances at the hitting distance in these two tasks (open bars of $\mathrm{H}$ and $\mathrm{R}$ ) were not significantly different $(p>0.3)$.

Furthermore, in the $\mathrm{R} 2$ task, the positional variance in the $x$ direction at the point equivalent to the hitting distance was significantly larger (intersubject paired $t$ test; $p<0.01$ ) than that in the $\mathrm{H}$ task. This also suggests that the follow-through after hitting is not a kind of reaching movements in which a target representation might exist. Because a clear MFR was observed during such a targetless condition, we conclude that the MFR can occur in the absence of either an explicit or implicit target representation.

\section{Modulation of the MFR without change in visual stimulus}

In the next experiment (experiment 6), we applied constant bias forces to the hand orthogonally to or against the reaching movement ( $+x,-x$, and $-y$ directions). Importantly, such force was independent of the hand movement (position, velocity, and acceleration), and visual stimulation was identical across all conditions. The subject was asked to make the same trajectory as in the nonbias force condition. Thus, if the MFR were generated by a motor command replanned based on the continuously updated position of the target representation affected by visual motion, we would expect the same corrective movement regardless of the
A

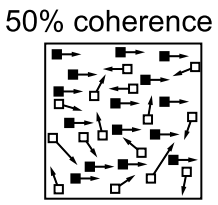

$30 \%$ coherence
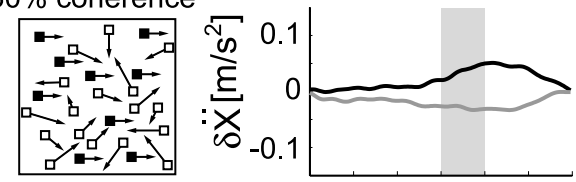

$10 \%$ coherence
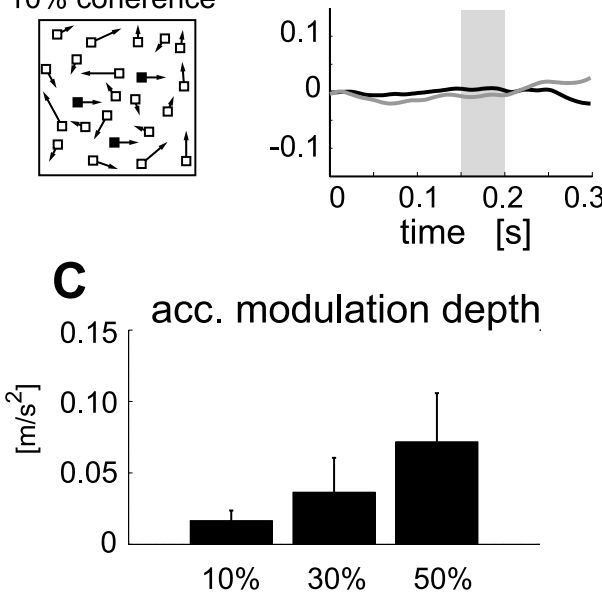

Figure 7. Responses to short lifetime coherent visual motion. $A$, Diagrams of the three visualmotion stimuli. Fifty percent, $30 \%$, and $10 \%$ of all dots shown as filled squares moved coherently, and remaining dots shown as white squares moved randomly. $\boldsymbol{B}$, Averaged hand $x$-acceleration responses to these stimuli in a typical subject. All data are presented relative to the control (no visual-motion). Black line, Response to the rightward visual motion; gray line, response to the leftward visual motion. C, Acceleration (acc.) modulation depth (mean difference accelerations to the rightward and leftward stimuli between 150 and $200 \mathrm{~ms}$ indicated by shaded area in $\boldsymbol{B}$ ) plotted against motion coherence. Each bar denotes the SD across four subjects. The modulation depth significantly increased with motion coherence $(r=0.72 ; t$ test; $p<0.01)$.

bias force. This expectation is supported by the observation that the movement pattern of target reaching in the force field becomes similar to that in free space after practice (Shadmehr and Mussa-Ivaldi, 1994). We found, however, that the acceleration modulation of the MFR increased with absolute bias force. Figure $9 A$ shows acceleration temporal patterns during $x$-directional positive bias-force tasks for a particular subject, in which the response amplitude increased with the bias force. Figure 9, $B$ and $C$, shows the acceleration modulation depth defined above for all bias force tasks. The linear regression analysis for each subject revealed that the slope between the absolute bias forces and response magnitudes was significant $(p<0.05)$ in all bias force

Table 2. Shifts in the remembered target position after the rightward (R) or leftward (L) visual motion

\begin{tabular}{lrrl}
\hline & \multicolumn{2}{l}{ Positional shift by visual motion $(\mathrm{cm})$} & \\
\cline { 2 - 3 } Subject & $\mathrm{L}$ & p value by $t$ test \\
\hline C & $0.32(0.74)$ & $0.16(0.98)$ & 0.63 \\
D & $-0.19(1.10)$ & $0.05(1.36)$ & 0.61 \\
E & $0.31(0.81)$ & $0.45(0.67)$ & 0.63 \\
F & $-0.36(1.04)$ & $-0.23(1.01)$ & 0.73 \\
\hline
\end{tabular}

The bias (mean position in control condition) was subtracted from the position in each subject. The values in parentheses denote SDs $(n=20)$. L, Leftward; R, rightward. 
A

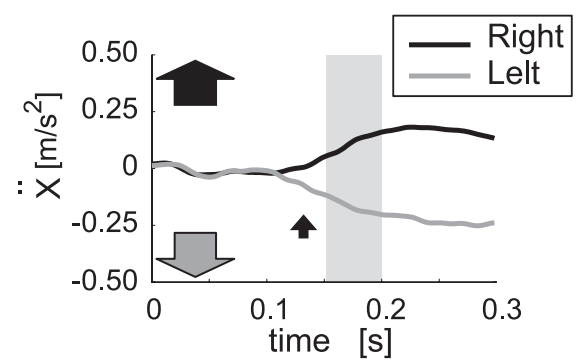

B

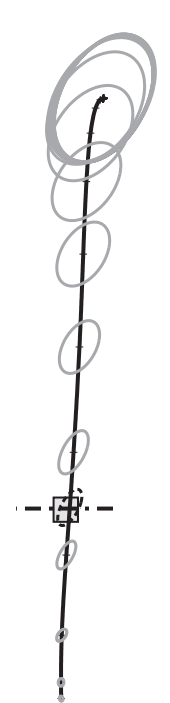

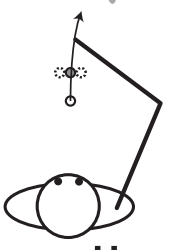

H

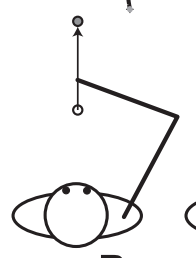

$\mathbf{R}$
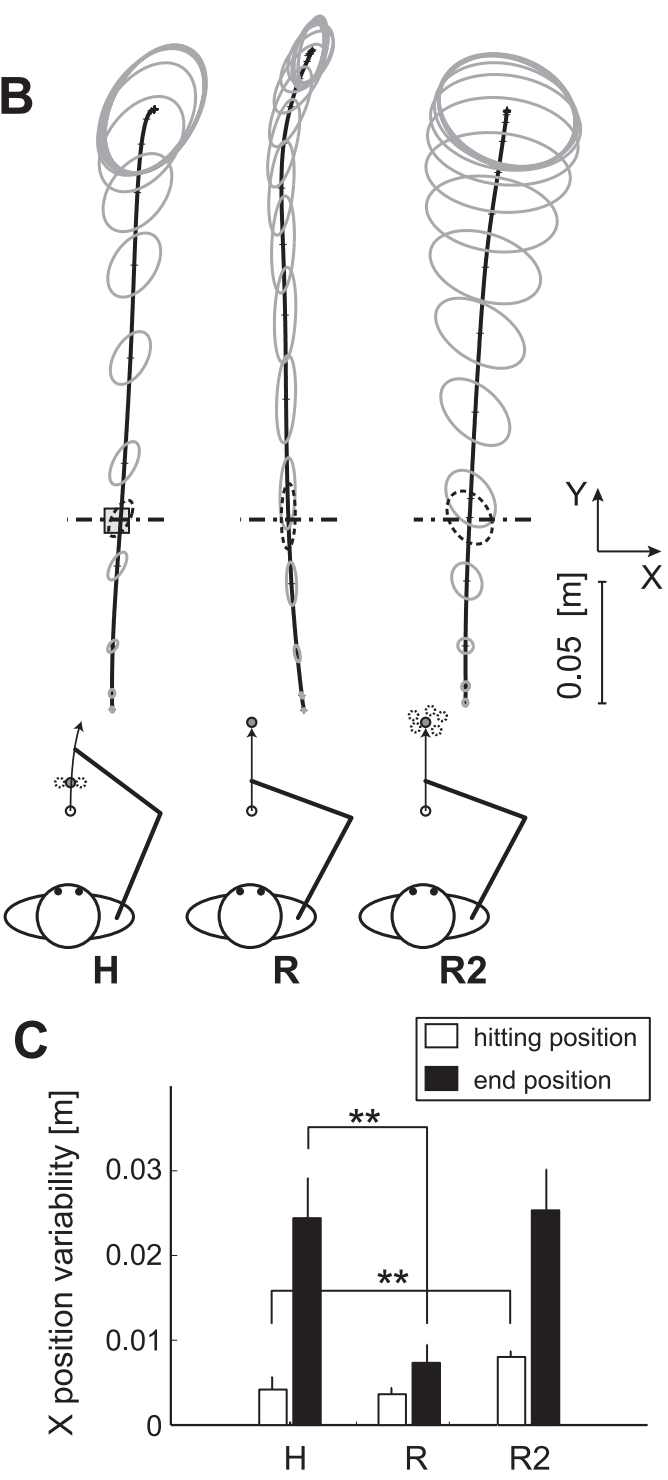

Figure 8. MFR during follow through in hitting task. A, Averaged $x$ accelerations of the trials with rightward and leftward visual motions applied during the follow-through phase in the $\mathrm{H}$ task of a typical subject. Offsets $(0-50 \mathrm{~ms})$ were subtracted from these responses. $\boldsymbol{B}$, Control trajectory variations in hitting task, reaching task for a fixed target, and reaching task for a randomly positioned target. The thick black line denotes averaged hand trajectories, and gray ellipses denote trajectory variances at every $100 \mathrm{~ms}$. The gray open square mark on the trajectory in the $\mathrm{H}$ task denotes the hitting target. The dotted ellipse in each graph denotes the hand positional variances at a particular $y$-directional distance $(y=0.38 \mathrm{~m}$ indicated by dash-dotted line). The diagram explaining each task is attached below each graph. $C, x$-positional variabilities (SD) at the hitting target distance ( $y=0.38 \mathrm{~m}$ ) (open bars) and at the movement end (filled bars) in each task. The double asterisks denote significant difference $(p<0.01)$ by paired $t$ test $(n=5)$.
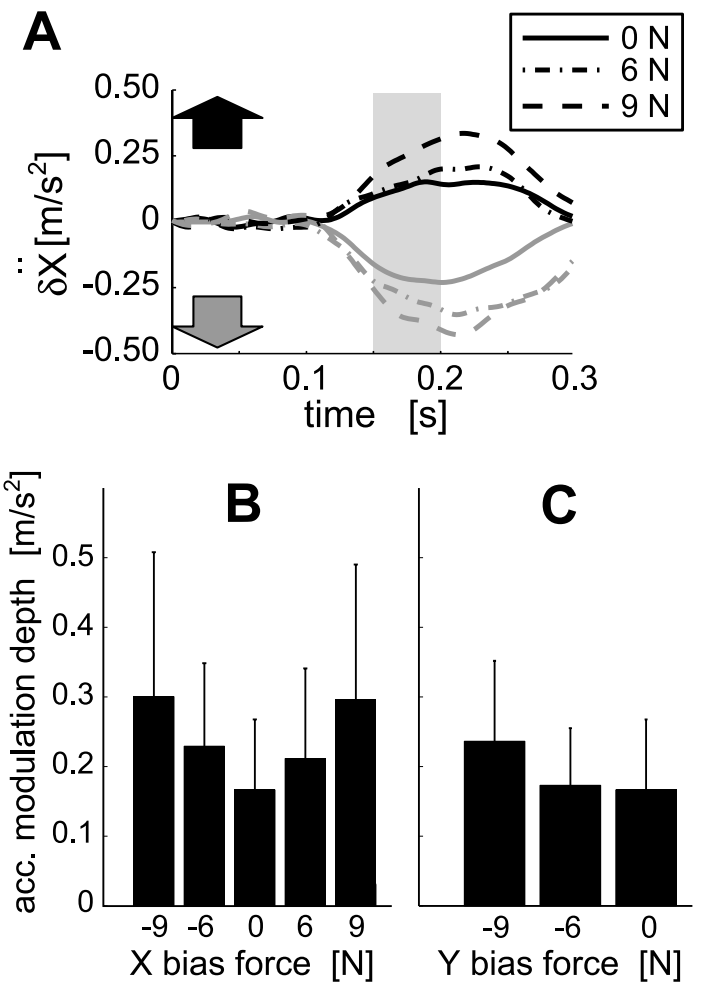

Figure 9. Effect of hand bias force on the MFR. $A$, Temporal patterns of acceleration responses for the rightward (black) and leftward (gray) stimuli in three $x$-bias force conditions $(0,6,9 \mathrm{~N})$. B, Acceleration (acc.) modulation depth (see Results) plotted against bias force applied in the direction orthogonal to the movement direction. C, Acceleration modulation depth plotted against bias force applied against movement direction. Error bars denote the SD across four subjects.

directions $(+x,-x,-y)$. This means that different corrective movements are initiated for the same visual motion condition.

Additionally, as has been found for somatosensory reflexes (Marsden et al., 1972), there were high correlations between the background EMGs elevated by the bias force and the EMG responses caused by visual motion $(r=0.71 \pm 0.30$ for the shoulder flexor; $r=0.95 \pm 0.03$ for the shoulder extensor). The motor command for the MFR may thus be organized reflexively, unlike the command planned for target reaching. The MFR amplitudes might be affected by the changes in corticospinal excitability (Hess et al., 1987; Mazzocchio et al., 1994) associated with concurrent feedforward motor commands.

\section{Discussion}

MFR initiation mechanism

We have described a rapid manual response induced by a largefield visual motion that occurs while the subject is making an arm reaching movement to an invisible target and examined its underlying mechanism in a series of behavioral experiments. Visual motion induces involuntary postural and eye movements as mentioned in the Introduction, and motor controls of individual body elements have many relationships with each other because of their many functional and dynamical interactions. Thus, we considered the possibility that the MFR is indirectly caused by visual motion through postural deviation or ocular response.

As described in the Results, however, the visual motion direction did not influence the EMGs of cervical and back muscles, which are usually involved in reflexive postural control (Keshner et al., 1988; Henry et al., 1998). This may be ascribed to the less activation of these muscles (Table 1) when the subject's body and chin are stabilized by a seat belt and supporting device, respec- 
tively. Therefore, we can exclude the possibility that the MFR is merely a byproduct of axial postural control. However, this does not exclude the possibility that the MFR might functionally act as an arm adjustment during postural fluctuation.

Recent eye-hand coordination studies (Prablanc et al., 1979; Herman and Maulucci, 1981; Biguer et al., 1982; Fischer and Rogal, 1986; Henriques et al., 1998, 2003; Turrell et al., 1998; Engel et al., 2000; Soechting et al., 2001; Ariff et al., 2002) suggest a couple of possibilities that the MFR could be affected by the OFR, both of which were indeed elicited by visual motion in our experimental setup.

One possibility is that the MFR reflects an error in manual pointing to a remembered target as observed when the subject shifts the gaze from the target position to the periphery (Henriques et al., 1998, 2003). This error, however, occurs in the direction opposite to the gaze shift, whereas the MFR occurs in the same direction as the OFR. The second possibility is that an efference copy of the ocular motor command drives the MFR. The latency of the rEMG of the shoulder muscle driving the MFR was nearly the same as that of the OFR (Fig. 3): the latency differences in the three subjects were $1,-1,-4 \mathrm{~ms}$, respectively. These latency differences are, however, much shorter than the time differences between the ocular movement and hand movement reported in previous studies (Turrell et al., 1998; Engel et al., 2000; Soechting et al., 2001), in which efference copy of the ocular motor command is deemed to be used as an arm motor command. Moreover, considering the transmission time from the motor cortex to the arm muscles [11-12 ms for biceps brachii, 14-15 ms for the brachioradialis, observed by transcranial magnetic stimulation (Abbruzzese et al., 1994)] and the delay from the oculomotor neuron activity to the eye movement [6-7 ms, which is estimated from Shidara and Kawano (1993) and Gomi et al. (1998)], the motor command for the MFR would be generated simultaneously with that for the OFR or even earlier.

This idea of the parallel motor command generation would also be supported by the difference in amplitude changes in the MFR and OFR shown in Figure 4. Especially, by introducing the fixation spot and stimulus-size reduction, the OFR almost disappeared, whereas the MFR was clearly observed. Therefore, it is unlikely that the MFR is attributable to the motor output of the oculomotor system. It is rather suggested that the information driving the MFR branches off in the earlier stages of the visual motion processing. Note that the latency of the OFR measured here $(86-89 \mathrm{~ms})$ was slightly longer than the values reported in the previous studies: 75-85 ms for humans (Gellman et al., 1990; Masson et al., 2000). This may be a result of the experimental setup, because the latency of the OFR is greatly dependent on the experimental condition (stimulus size, contrast, spatiotemporal structure, and postsaccadic delay) as described by Kawano and Miles (1986), Miles et al. (1986), and Gellman et al. (1990).

\section{Effects of visual motion on target and hand positions for MFR initiation}

Could the MFR arise from effects of visual motion on target or hand position representations? Several studies have shown that the surrounding visual motion induces an apparent motion of the visual target in the opposite direction (the "induced motion" illusion), resulting in saccadic and pointing errors in the direction opposite to visual motion (Zivotofsky et al., 1996; Soechting et al., 2001). However, the MFR cannot be explained by the induced motion of the remembered target, because the motor response follows, rather than opposes, the background motion.

Another possibility suggested by recent studies (Brenner and
Smeets, 1997; Whitney et al., 2003) is that visual motion induces a drift in the represented target position in the visual motion direction, and then that drift produces a quick arm response. Real target shifts indeed elicit rapid reaching adjustments (Goodale et al., 1986; Prablanc and Martin, 1992; Pisella et al., 1998, 2000; Day and Lyon, 2000; Desmurget and Grafton, 2000; Brouwer et al., 2002; Johnson and Haggard, 2005) whose latency is comparable with that evoked by the large-field visual motion. If target representation is affected by the visual motion, a rapid response could occur. Interestingly, in previous studies, when the visual motion and visible reaching target were presented together (Brenner and Smeets, 1997; Whitney et al., 2003), perceptual mislocalization of the target was observed. In our study, the reaching target and the large-field visual motion were presented at different times, and we found no evidence of target mislocalization (Table 2). Furthermore, we demonstrated that the MFR was still present during the follow-through movement after the target was hit. The kinematic analysis suggested that the actual trajectory of follow-through movement cannot be regarded as part of a reaching movement and that it was effectively targetless. The quick manual response we observed during the followthrough movement, therefore, cannot be explained in terms of target positional shift. We thus conclude that the MFR is not related to representations of target location.

Could the MFR arise from an indirect effect of visual motion on a neural representation of hand position? We might assume that visual motion generates an apparent motion of the represented hand position opposite to the large-field visual motion as in the induced-motion illusions. A corrective movement could then occur in response to the illusory apparent hand motion. The direction of this correction would then coincide with that of the large-field visual motion. Indeed, it has been reported that perturbation of the hand position information in visual feedback induces such a corrective response in arm movement (Saunders and Knill, 2003). As far as we know, however, the latency of the corrective manual response to the perturbation on the visual feedback of the hand position [ $\sim 160 \mathrm{~ms}$ (Saunders and Knill, 2003)] is longer than the latency of the MFR (126 ms) but is comparable with that of the voluntary reaction demonstrated in Figure $2 B$. In addition, the subject's hand in our experiment was always occluded by the screen, and, in all experiments, its location marker was not shown during arm movements. This setup would prevent generating a quick corrective response to the perturbed information for the hand position because the reaction is greatly delayed when the hand position is invisible (van Donkelaar et al., 1994). In light of these examinations, it is unlikely that the MFR is a reaction to the representation of the hand position affected by the surrounding visual motion.

\section{Cortical computational mechanisms for generating the MFR}

As we described previously, the MFR shares many phenomenal properties with the OFR, a reflexive ocular following response induced by visual motion. We therefore speculate that the underlying neural mechanisms of the MFR may considerably overlap with those of the OFR. The neural control of the OFR involves the middle temporal area (MT) and medial superior temporal area (MST) (Kawano et al., 1994), which encode various types of visual motion signals (Duffy and Wurtz, 1991, 1997; Britten et al., 1993; Movshon and Newsome, 1996). According to several electrophysiological studies on monkeys, the visual motion signals encoded in the MST are sent to the vestibular nuclei via the dorsolateral pontine nucleus (DLPN) (Kawano et al., 1992) and the ventral paraflocculus in the cerebellum (Shidara et al., 1993; 
Gomi et al., 1998; Takemura et al., 2001). Here, visual motion signals are transformed into the ocular motor commands in terms of eye muscle coordination (kinematics) and the temporal profile (dynamics).

For arm control, on the other hand, visual motion signals have to be transformed into motor commands for arm muscles, not for ocular muscles. We infer that the visual motion information represented in the areas MT, MST, and DLPN areas could be shared for producing the ocular and manual responses but that such information might be separately sent to the command generation processes of each motor system. A possible hypothesis is that the intermediate and lateral cerebellum regions involved in visually guided arm control (Coltz et al., 1999; Liu et al., 2003) contributes to the generation of arm muscle motor commands for the MFR. The neural pathway for the MFR would be at least partly independent from the mechanism for generating rapid reaching adjustment: the latter depends on computations of pointing position error in the posterior parietal cortex (Desmurget and Grafton, 2000; Pisella et al., 2000; Buneo et al., 2002). Because background motion has also been reported to affect the target localization (Brenner and Smeets, 1997; Whitney et al., 2003), however, these two mechanisms (target coding and visual motion coding) could also interact with each other. Additional psychophysical and physiological studies would clarify which computations are shared and which are separate in visuomotor processing using the information of target localization and background motion.

The above observations and examinations all together suggest that visual motion signals are directly transformed into manual motor commands in a special reflexive pathway. Because of this parallel processing, the motor commands generating the MFR would be affected by the voluntary motor commands (i.e., background EMG; see Results) in the same way as somatosensory reflex processing (Marsden et al., 1972). Besides the preplanned feedforward control and target-based feedback control, we need to further investigate the basic mechanism of visuomotor control probed by the short-latency MFR that could potentially contribute to reduce the effective latency of voluntary reaction.

\section{References}

Abbruzzese G, Morena M, Spadavecchia L, Schieppati M (1994) Response of arm flexor muscles to magnetic and electrical brain stimulation during shortening and lengthening tasks in man. J Physiol (Lond) 481:499-507.

Alexander GE, Crutcher MD (1990) Preparation for movement: neural representations of intended direction in three motor areas of the monkey. J Neurophysiol 64:133-150.

Ariff G, Donchin O, Nanayakkara T, Shadmehr R (2002) A real-time state predictor in motor control: study of saccadic eye movements during unseen reaching movements. J Neurosci 22:7721-7729.

Biguer B, Jeannerod M, Prablanc C (1982) The coordination of eye, head, and arm movements during reaching at a single visual target. Exp Brain Res 46:301-304.

Blouin J, Gauthier GM, Vercher JL (1995) Failure to update the egocentric representation of the visual space through labyrinthine signal. Brain Cogn 29:1-22.

Blouin J, Okada T, Wolsley C, Bronstein A (1998) Encoding target-trunk relative position: cervical versus vestibular contribution. Exp Brain Res 122:101-107.

Braddick OJ (1980) Low-level and high-level processes in apparent motion. Philos Trans R Soc Lond B Biol Sci 290:137-151.

Brenner E, Smeets JB (1997) Fast responses of the human hand to changes in target position. J Mot Behav 29:297-310.

Britten KH, Shadlen MN, Newsome WT, Movshon JA (1993) Responses of neurons in macaque MT to stochastic motion signals. Vis Neurosci 10:1157-1169.

Brouwer AM, Brenner E, Smeets JJ (2002) Hitting moving objects: is target speed used in guiding the hand? Exp Brain Res 143:198-211.
Buneo CA, Jarvis MR, Batista AP, Andersen RA (2002) Direct visuomotor transformations for reaching. Nature 416:632-636.

Caminiti R, Johnson PB, Ferraina S, Burnod Y (1992) Reaching toward visual targets. I. Neurophysiological studies. In: Control of arm movement in space (Caminiti R, Johnson PB, Burnod Y, eds), pp 147-183. Berlin: Springer.

Coltz JD, Johnson MT, Ebner TJ (1999) Cerebellar Purkinje cell simple spike discharge encodes movement velocity in primates during visuomotor arm tracking. J Neurosci 19:1782-1803.

Day BL, Lyon IN (2000) Voluntary modification of automatic arm movements evoked by motion of a visual target. Exp Brain Res 130:159-168.

Desmurget M, Grafton S (2000) Forward modeling allows feedback control for fast reaching movements. Trends Cogn Sci 4:423-431.

Duffy CJ, Wurtz RH (1991) Sensitivity of MST neurons to optic flow stimuli. II. Mechanisms of response selectivity revealed by small-field stimuli. J Neurophysiol 65:1346-1359.

Duffy CJ, Wurtz RH (1997) Medial superior temporal area neurons respond to speed patterns in optic flow. J Neurosci 17:2839-2851.

Engel KC, Anderson JH, Soechting JF (2000) Similarity in the response of smooth pursuit and manual tracking to a change in the direction of target motion. J Neurophysiol 84:1149-1156.

Fischer B, Rogal L (1986) Eye-hand-coordination in man: a reaction time study. Biol Cybern 55:253-261.

Gellman RS, Carl JR, Miles FA (1990) Short latency ocular-following responses in man. Vis Neurosci 5:107-122.

Georgopoulos AP (1995) Current issues in directional motor control. Trends Neurosci 18:506-510.

Ghez C, Krakauer J (1991) The organization of movement. In: Principles of neural science, Ed 4 (Kandel ER, Schwartz JH, Jessell TM, eds), pp 653673. New York: McGraw-Hill.

Gomi H, Kawato M (1996) Equilibrium-point control hypothesis examined by measured arm-stiffness during multi-joint movement. Science 272:117-120.

Gomi H, Shidara M, Takemura A, Inoue Y, Kawano K, Kawato M (1998) Temporal firing patterns of Purkinje cells in the cerebellar ventral paraflocculus during ocular following responses in monkeys I. Simple spikes. J Neurophysiol 80:818-831.

Goodale MA, Pelisson D, Prablanc C (1986) Large adjustments in visually guided reaching do not depend on vision of the hand or perception of target displacement. Nature 320:748-750.

Henriques DY, Klier EM, Smith MA, Lowy D, Crawford JD (1998) Gazecentered remapping of remembered visual space in an open-loop pointing task. J Neurosci 18:1583-1594.

Henriques DY, Medendorp WP, Gielen CC, Crawford JD (2003) Geometric computations underlying eye-hand coordination: orientations of the two eyes and the head. Exp Brain Res 152:70-78.

Henry SM, Fung J, Horak FB (1998) EMG responses to maintain stance during multidirectional surface translations. J Neurophysiol 80:1939-1950.

Herman R, Maulucci R (1981) Visually triggered eye-arm movements in man. Exp Brain Res 42:392-398.

Hess CW, Mills KR, Murray NM (1987) Responses in small hand muscles from magnetic stimulation of the human brain. J Physiol (Lond) 388:397-419.

Ito T, Murano EZ, Gomi H (2004) Fast force-generation dynamics of human articulatory muscles. J Appl Physiol 96:2318-2324.

Johnson H, Haggard P (2005) Motor awareness without perceptual awareness. Neuropsychologia 43:227-237.

Kakei S, Hoffman DS, Strick PL (1999) Muscle and movement representations in the primary motor cortex. Science 285:2136-2139.

Kalaska JF, Crammond DJ, Cohen DAD, Prud'homme M, Hyde ML (1992) Comparison of cell discharge in motor, premotor, and parietal cortex during reaching. In: Control of arm movement in space (Caminiti R, Johnson PB, Burnod Y, eds), pp 129-146. Berlin: Springer.

Kawano K, Miles FA (1986) Short-latency ocular following responses of monkey. II. Dependence on a prior saccadic eye movement. J Neurophysiol 56:1355-1380.

Kawano K, Shidara M, Yamane S (1992) Neural activity in dorsolateral pontine nucleus of alert monkey during ocular following response. J Neurophysiol 67:680-703.

Kawano K, Shidara M, Watanabe Y, Yamane S (1994) Neural activity in cortical area MST of alert monkey during ocular following responses. J Neurophysiol 71:2305-2324. 
Kawato M (1999) Internal models for motor control and trajectory planning. Curr Opin Neurobiol 9:718-727.

Keshner EA, Woollacott MH, Debu B (1988) Neck, trunk and limb muscle responses during postural perturbations in humans. Exp Brain Res 71:455-466.

Liu X, Robertson E, Miall RC (2003) Neuronal activity related to the visual representation of arm movements in the lateral cerebellar cortex. J Neurophysiol 89:1223-1237.

Marsden C, Merton P, Morton H (1972) Servo action in human voluntary movement. Nature 238:140-143.

Masson GS, Rybarczyk Y, Castet E, Mestre DR (2000) Temporal dynamics of motion integration for the initiation of tracking eye movements at ultra-short latencies. Vis Neurosci 17:753-767.

Mazzocchio R, Rothwell JC, Day BL, Thompson PD (1994) Effect of tonic voluntary activity on the excitability of human motor cortex. J Physiol (Lond) 474:261-267.

Miles FA, Kawano K (1986) Short-latency ocular following responses of monkey. III. Plasticity. J Neurophysiol 56:1381-1396.

Miles FA, Kawano K, Optican LM (1986) Short-latency ocular following responses of monkey. I. Dependence on temporospatial properties of visual input. J Neurophysiol 56:1321-1354.

Movshon JA, Newsome WT (1996) Visual response properties of striate cortical neurons projecting to area MT in macaque monkeys. J Neurosci 16:7733-7741.

Murakami I, Shimojo S (1993) Motion capture changes to induced motion at higher luminance contrasts, smaller eccentricities, and larger inducer sizes. Vision Res 33:2091-2107.

Nakayama K, Tyler CW (1981) Psychophysical isolation of movement sensitivity by removal of familiar position cues. Vision Res 21:427-433.

Nashner LM, Berthoz A (1978) Visual contribution to rapid motor responses during postural control. Brain Res 150:403-407.

Newsome WT, Pare EB (1988) A selective impairment of motion perception following lesions of the middle temporal visual area (MT). J Neurosci 8:2201-2211.

Nishida S, Johnston A (1999) Influence of motion signals on the perceived position of spatial pattern. Nature 397:610-612.

Pisella L, Arzi M, Rossetti Y (1998) The timing of color and location processing in the motor context. Exp Brain Res 121:270-276.

Pisella L, Grea H, Tilikete C, Vighetto A, Desmurget M, Rode G, Boisson D, Rossetti Y (2000) An "automatic pilot" for the hand in human posterior parietal cortex: toward reinterpreting optic ataxia. Nat Neurosci 3:729-736.
Prablanc C, Martin O (1992) Automatic control during hand reaching at undetected two-dimensional target displacements. J Neurophysiol 67:455-469.

Prablanc C, Echallier JF, Komilis E, Jeannerod M (1979) Optimal response of eye and hand motor systems in pointing at a visual target. I. Spatiotemporal characteristics of eye and hand movements and their relationships when varying the amount of visual information. Biol Cybern 35:113-124.

Ramachandran VS (1987) Interaction between colour and motion in human vision. Nature 328:645-647.

Saunders JA, Knill DC (2003) Humans use continuous visual feedback from the hand to control fast reaching movements. Exp Brain Res 152:341-352.

Shadmehr R, Mussa-Ivaldi FA (1994) Adaptive representation of dynamics during learning of a motor task. J Neurosci 14:3208-3224.

Shidara M, Kawano K (1993) Role of Purkinje cells in the ventral paraflocculus in short-latency ocular following responses. Exp Brain Res 93:185-195.

Shidara M, Kawano K, Gomi H, Kawato M (1993) Inverse dynamics model eye movement control by Purkinje cells in the cerebellum. Nature 365:50-52.

Soechting JF, Engel KC, Flanders M (2001) The Duncker illusion and eyehand coordination. J Neurophysiol 85:843-854.

Takemura A, Inoue Y, Gomi H, Kawato M, Kawano K (2001) Change in neuronal firing patterns in the process of motor command generation for the ocular following responses. J Neurophysiol 86:1750-1763.

Turrell Y, Bard C, Fleury M, Teasdale N, Martin O (1998) Corrective loops involved in fast aiming movements: effect of task and environment. Exp Brain Res 120:41-51.

van de Grind WA, Koenderink JJ, van Doorn AJ (1986) The distribution of human motion detector properties in the monocular visual field. Vision Res 26:797-810.

van Donkelaar P, Lee RG, Gellman RS (1994) The contribution of retinal and extraretinal signals to manual tracking movements. Exp Brain Res 99:155-163.

Whitney D, Cavanagh P (2000) Motion distorts visual space: shifting the perceived position of remote stationary objects. Nat Neurosci 3:954-959.

Whitney D, Westwood DA, Goodale MA (2003) The influence of visual motion on fast reaching movements to a stationary object. Nature 423:869-873.

Wolpert DM, Ghahramani Z (2000) Computational principles of movement neuroscience. Nat Neurosci [Suppl] 3:1212-1217.

Zivotofsky AZ, Rottach KG, Averbuch-Heller L, Kori AA, Thomas CW, Dell'Osso LF, Leigh RJ (1996) Saccades to remembered targets: the effects of smooth pursuit and illusory stimulus motion. J Neurophysiol 76:3617-3632. 